\title{
A Wavelet Based Image Retrieval
}

\author{
Kalyani Mali ${ }^{1}$ and Rana Datta Gupta ${ }^{2}$ \\ 1 Department of Computer Science, Kalyani University, \\ Kalyani 741 235, India \\ 2 Department of Computer Science \& Engineering, Jadavpur University, \\ Calcutta, India \\ kalyani_mali@yahoo.com, rdg@ieee.org
}

\begin{abstract}
An wavelet based image retrieval scheme is described. Wavelet transforms are applied to compress the image space, thereby reducing noise. A combination of texture, shape, topology and fuzzy geometric features, that is invariant to orientation, scale and object deformation, are extracted from this compressed image. The use of wavelet based compression eliminates the need for any other preprocessing. The extracted features serve as the signature of the compressed images, in terms of their content. Their use in content based image retrieval is demonstrated.
\end{abstract}

Keywords: Image mining, wavelets, content based image retrieval, image compression.

\section{Introduction}

Wavelets [1] are found to be very useful in appropriately modeling the nonstationary property of image signals formed around the edges, as well as the correlation amongst the image pixels. The role of wavelets in different aspects of data mining is gaining significant importance, and it has become a very powerful signal processing tool in different application areas such as image processing, compression, image indexing and retrieval, digital libraries, image clustering and databases [2, 3]. Most of the activities in mining image data have been in the search and retrieval of images based on the analysis of similarity of a query image or its feature(s) with the entries in the image database. In Content Based Image Retrieval (CBIR) systems, the images are searched and retrieved by extracting suitable features based on the visual content of the images [4,5]. CBIR has increasingly become a growing area of study towards the successful development of image mining techniques.

In this article we describe a wavelet based CBIR on real life digital images. Wavelet transforms are applied to compress the image space, thereby eliminating noise. The processing now needs to be done on subsampled images of smaller size. This also enables us to eliminate the need for any other preprocessing of the raw images. A combination of texture, shape, topology and fuzzy geometric features are extracted directly from this compressed image. The features are next used for CBIR in the compressed domain. The images retrieved, based on content, are always found to lie in the same partition as the query image. 


\section{Wavelet Transform}

Wavelet transform is a signal processing technique that decomposes a signal or image into different frequency subbands at number of levels and multiple resolutions. In every level of decomposition, the high-frequency subband captures the discontinuities in the signals - for example, the edge information in an image. The low-frequency subband is a subsampled version of the original image, with similar statistical and spatial properties as the original signal. As a result, the low-frequency subband can be further decomposed into higher levels of resolution, and it helps in representing spatial objects in different coarser levels of accuracy in multiresolution subbands. The wavelet transform is typically represented as a pair of high-pass and low-pass filters, with many wavelet basis functions being available in literature [1].

Haar wavelet is one of the simplest wavelet. Daubechies wavelet family corresponds to a set of compactly supported orthonormal wavelets. These are denoted as $\operatorname{db} N$, where $N$ stands for the order of the wavelet. In this article we focus on Daubechies family of wavelets $\mathrm{db}-N(N=1,2,3,4,5,6)$ as well as db-9/7 [6]. The db- 1 wavelet is the same as the Haar function.

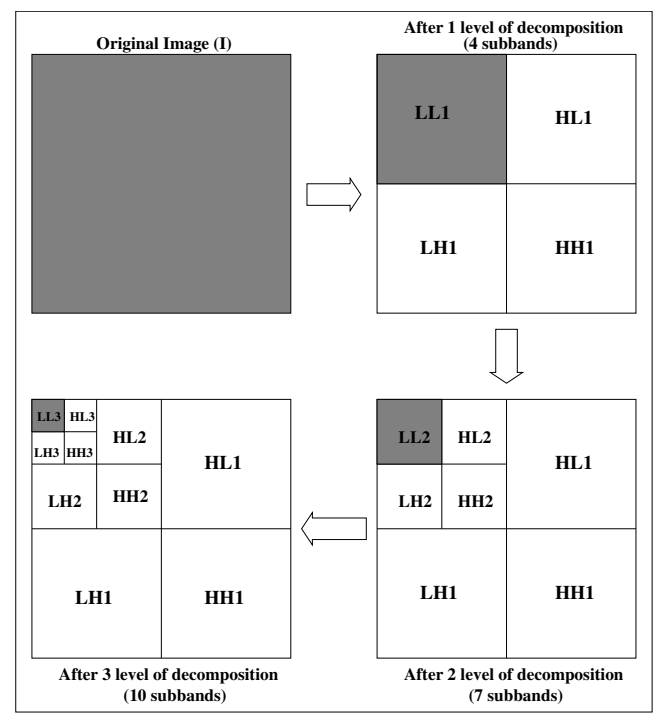

Fig. 1. Three level multiresolution wavelet decomposition of an image

In Fig. 1, we show an example of hierarchical wavelet decomposition of an image into ten subbands after three levels of decomposition [4. After the first level of decomposition, the original image is decomposed into four subbands $L L 1$, $H L 1, L H 1$, and $H H 1$. Since the low-frequency subband $L L 1$ has similar spatial and statistical characteristics as the original image, it can be further decomposed into four subbands $L L 2, H L 2, L H 2$, and $H H 2$. 


\section{$3 \quad$ Feature Extraction}

In this section we mention the input features used. These include texture, fuzzy geometry, moment invariants and Euler vector. They are useful in characterizing images, and can be used as a signature of image content. Hence these features have promising application in CBIR.

\subsection{Texture}

Texture is one of the important features used in identifying objects or regions of interest in an image [7]. The contextual texture information is specified by the matrix of relative frequencies $P(i, j)$ with which two neighboring resolution cells, having gray levels $i$ and $j$ and separated by a distance $\delta$, occur in the image. The angular second moment, inverse difference moment, Contrast, Entropy of the image are calculated four times, corresponding to each of the four directional cooccurrence matrices.

\subsection{Shape}

Shape can roughly be defined as the description of an object minus its position, orientation and size. Therefore, shape features should be invariant to translation, rotation, and scale, when the arrangement of the objects in the image are not known in advance.

Two of the seven transformation invariant moments, used here, are expressed as follows.

$$
\left.\begin{array}{l}
\phi_{1}=\left(\eta_{2,0}+\eta_{0,2}\right) \\
\phi_{2}=\left(\eta_{2,0}-\eta_{0,2}\right)^{2}+4 \eta_{1,1}^{2}
\end{array}\right\}
$$

\subsection{Topology}

One topological property of a digital image is known as Euler number. Although typically computed in a binary image, it can be extended to characterize graytone images by defining the Euler Vector [8]. If an image has $C$ connected components and $H$ number of holes, the Euler number $E$ of the image can be defined as $E=C-H$.

Intensity value of each pixel in an 8-bit gray-tone image can be represented by an 8 -bit binary Euler vector $b_{i}, i=0,1, \cdots, 7$. The $i$ th bit plane is formed with $b_{i}$ 's from all the pixels in the gray-tone image. We retain the first two most significant bit planes corresponding to $\left(b_{7}, b_{6}\right)$, because they contain most of the information of the image.

\subsection{Fuzzy Geometry}

A fuzzy subset of a set $S$ is a mapping $\mu$ from $S$ into $[0,1]$ and denotes the degree of membership of an object. The compactness (Comp) [9] of a fuzzy set $\mu$ having area $a(\mu)$ and perimeter $p(\mu)$ is defined as 


$$
\operatorname{Comp}(\mu)=\frac{a(\mu)}{p^{2}(\mu)}
$$

Here the area is defined as $a(\mu)=\sum \mu$, the summation being considered over a region outside which $\mu=0$. The perimeter of an image is expressed as $p(\mu)=$ $\sum_{i, j}|\mu(i)-\mu(j)|$, where $\mu(i)$ and $\mu(j)$ are the membership values of two adjacent pixels.

\section{Implementation and Results}

A Content Based Image Retrieval were performed on 86 images, consisting of five different image categories such as 13 aeroplanes, 22 cars, 41 flowers of two types and 10 zebras. A sample image is presented to the system for content based retrieval of the closest match.

Wavelet transform is applied,followed by feature extraction. Performance is evaluated with each of these Daubechies functions in the LL1, LL2, LL3 subbands. The query image is identified as belonging to the majority class among the five nearest objects retrieved. Table 1 quantitatively evaluates the CBIR performance using Daubechies family wavelets on the basis of correct classification.

Table 1. CBIR Performance using different Daubechies wavelet functions

\begin{tabular}{|c|c|c|c|c|c|c|c|}
\hline \multirow{2}{*}{\multicolumn{2}{|c|}{ Wavelet }} & \multicolumn{5}{|c|}{ Image category } & \multirow[t]{2}{*}{ Average } \\
\hline & & Aero & Car & Flow1 & Flow2 & Zebra & \\
\hline \multirow{3}{*}{ db1 } & LL1 & 15.38 & 63.64 & 58.82 & 50.00 & 60.00 & 49.57 \\
\hline & LL2 & 30.77 & 54.55 & 52.94 & 70.83 & 90.00 & 59.82 \\
\hline & \begin{tabular}{|l|} 
LL3 \\
\end{tabular} & 23.08 & 54.55 & 82.35 & 75.00 & 100.00 & 67.00 \\
\hline \multirow{3}{*}{$\mathrm{db} 2$} & LL1 & 46.15 & 50.00 & 52.95 & 75.00 & 60.00 & $\overline{56.82}$ \\
\hline & LL2 & 61.54 & 50.0 & 64.71 & 75.00 & 100.00 & 70.25 \\
\hline & \begin{tabular}{|l|} 
LL3 \\
\end{tabular} & 53.85 & 45.45 & 64.71 & 87.50 & 100.00 & 70.30 \\
\hline \multirow{3}{*}{ db3 } & LL1 & 38.46 & 59.09 & 58.82 & 79.17 & 60.00 & $\overline{59.11}$ \\
\hline & \begin{tabular}{|l|}
$\mathrm{LL} 2$ \\
\end{tabular} & 23.08 & 63.64 & 70.59 & 75.00 & 100.00 & 66.46 \\
\hline & \begin{tabular}{|l|} 
LL33 \\
\end{tabular} & 46.15 & 63.64 & 76.47 & 83.33 & 100.00 & $\overline{73.92}$ \\
\hline \multirow{3}{*}{$\mathrm{db} 4$} & LL1 & 46.15 & 45.45 & 70.59 & 75.00 & 80.00 & 63.44 \\
\hline & \begin{tabular}{|l|}
$\mathrm{LL} 2$ \\
\end{tabular} & 38.46 & 54.55 & 64.71 & 87.50 & 100.00 & 69.04 \\
\hline & LL3 & 38.46 & 68.18 & 47.06 & 83.33 & 70.00 & 61.41 \\
\hline \multirow{3}{*}{ db5 } & LL1 & 53.85 & 45.45 & 52.94 & 70.83 & 70.00 & 58.62 \\
\hline & $\overline{\mathrm{LL} 2}$ & 53.85 & 68.18 & 70.59 & 87.50 & 100.00 & $\overline{76.02}$ \\
\hline & LL3 & \begin{tabular}{|l|}
7.69 \\
\end{tabular} & 68.18 & 47.06 & 79.17 & 80.00 & $\overline{56.42}$ \\
\hline \multirow{3}{*}{ db6 } & LL1 & 61.54 & 59.09 & 52.94 & 70.83 & 70.00 & 62.88 \\
\hline & LL2 & 30.77 & 81.82 & 76.47 & 66.67 & 100.00 & 71.15 \\
\hline & LL3 & 15.38 & 54.55 & 58.82 & 83.33 & 100.00 & 62.42 \\
\hline \multirow{3}{*}{$\mathrm{db} 9 / 7$} & LL1 & 30.77 & 50.00 & 58.82 & 66.67 & 80.00 & 57.25 \\
\hline & LL2 & 30.77 & 40.91 & 70.59 & 75.00 & 100.00 & 63.45 \\
\hline & \begin{tabular}{|l|} 
LL3 \\
\end{tabular} & 53.85 & 45.45 & 70.59 & 83.30 & 100.00 & 70.64 \\
\hline \multicolumn{2}{|c|}{ original } & 15.38 & 77.27 & 64.71 & 62.50 & 90.00 & 61.97 \\
\hline
\end{tabular}



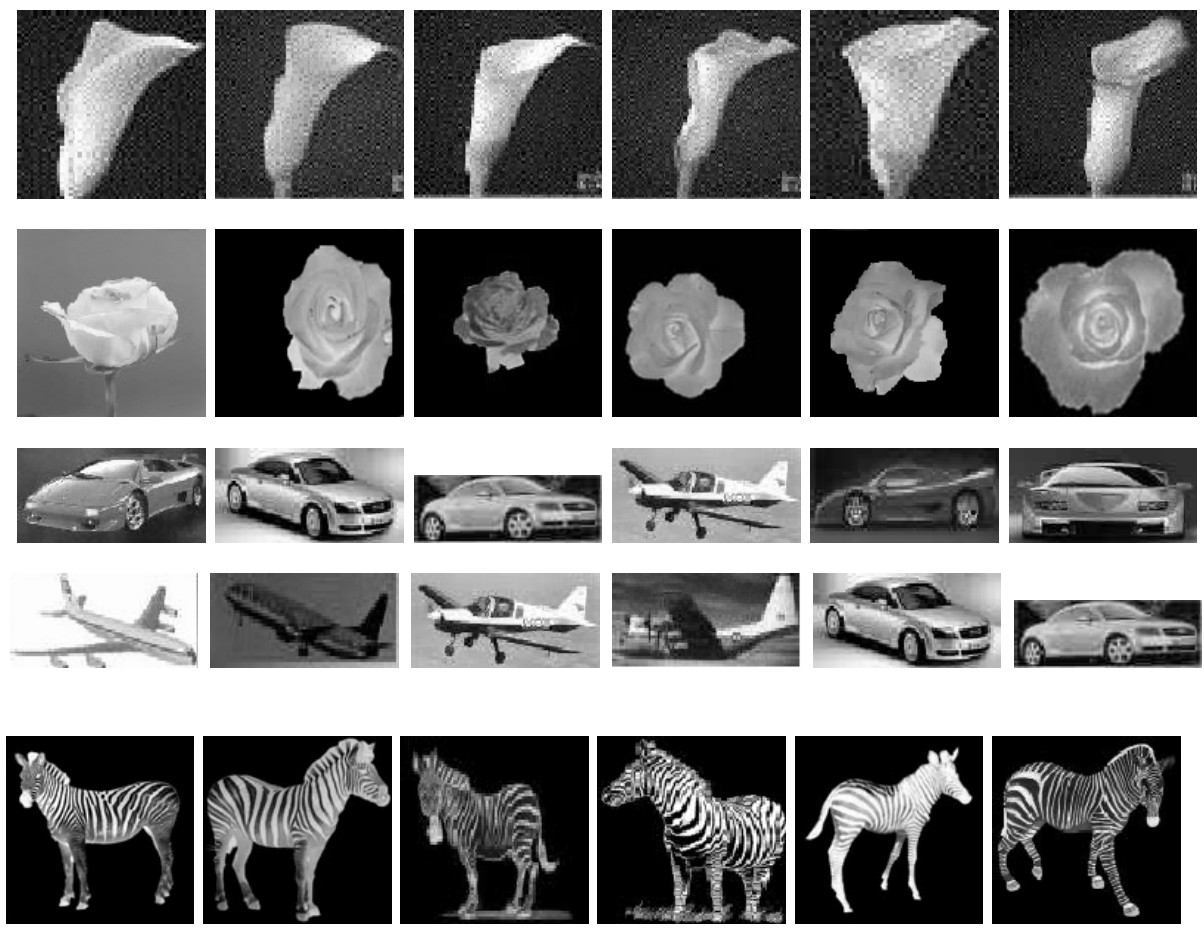

Fig. 2. CBIR for sample images in $L L 2$ subband using db-5 function

The performance is compared with the results obtained from the original image. It is shown that performance is generally better in the higher subbands. Fig. 2 demonstrates sample CBIR results, from the $L L 2$ subband of the wavelet decomposition, using db-5 function. The first column refers to the query image. The rest of the columns, along each row, depict the content-based retrieved images graded according to increasing distance from the corresponding query image. It is to be noted that most of the retrieved images are found to lie in the same subcategory as the query image.

On the whole, the results serve to highlight the utility of the extracted features, in the compressed domain, for CBIR.

\section{Conclusions and Discussion}

Wavelet transforms are employed to compress the image, and eliminate noise. This allows us to avoid the need for any other preprocessing of the raw image. The signature of the compressed images, in terms of their visual content, is extracted as features involving texture, shape, topology and fuzzy geometry. The features are invariant to orientation, scale and deformation of object, as well as the background intensity. 
Use of these features for CBIR, in the compressed domain, is demonstrated. The retrieved images are always found to lie in the same partition as that of the corresponding query image. Since the processing is done on subsampled (compressed) images of smaller size, this strategy now opens up interesting propositions for large scale image mining.

\section{References}

1. Daubechies, I.: Ten Lectures on Wavelets. CBMS, Philadelphia: Society for Industrial and Applied Mathematics (1992)

2. Li, T., Li, Q., Zhu, S., Ogihara, M.: A survey on wavelet applications in data mining. ACM SIGKDD Explorations Newsletter 4 (2002)

3. de Wouwer, G.V., Scheunders, P., Dyck, D.V.: Statistical texture characterization from discrete wavelet representations. IEEE Transactions on Image Processing $\mathbf{8}$ (1999) 592-598

4. Mitra, S., Acharya, T.: Data Mining: Multimedia, Soft Computing, and Bioinformatics. New York: John Wiley (2003)

5. Castelli, V., Bergman, L.: Image Databases: Search and Retrieval of Digital Imagery. New York: Wiley-Interscience (2001)

6. Daubechies, I.: The wavelet transform, time-frequency localization and signal analysis. IEEE Trans. Infor. Theory 36 (1990) 961-1005

7. Haralick, R.M., Shanmugam, K., Dinstein, I.: Textural features for image classification. IEEE Transactions on Systems, Man, and Cybernetics 3 (1973) 610-621

8. Bishnu, A., Bhattacharya, B.B., Kundu, M.K., Murthy, C.A., Acharya, T.: Euler vector: A combinatorial signature of gray-tone images. Proc. of the IEEE Int. Conf. on Information Technology: Coding \& Computing (2002)

9. Pal, S.K., Mitra, S.: Neuro-fuzzy Pattern Recognition: Methods in Soft Computing. New York: John Wiley (1999) 